Meta

Journal des traducteurs

Translators' Journal

\title{
Index des mots et sujets traités dans le volume 16
}

Volume 16, numéro 4, décembre 1971

URI : https://id.erudit.org/iderudit/003213ar

DOI : https://doi.org/10.7202/003213ar

Aller au sommaire du numéro

Éditeur(s)

Les Presses de l'Université de Montréal

ISSN

0026-0452 (imprimé)

1492-1421 (numérique)

Découvrir la revue

Citer ce document

(1971). Index des mots et sujets traités dans le volume 16. Meta, 16(4), 263-267.

https://doi.org/10.7202/003213ar d'utilisation que vous pouvez consulter en ligne.

https://apropos.erudit.org/fr/usagers/politique-dutilisation/ 


\section{INDEX DES MOTS ET SUJETS TRAITES DANS LE VOLUME 16}

Abandon de commission, 3, p. 171.

Abréviations (traitement des), 1-2, p. 128.

Accent de phrase en anglais, 1-2, p. 20.

Achat spontané / par impulsion, 4, p. 235.

Acquisition du vocabulaire scientifique, 1-2, p. 66.

Adaptation, 3, p. 153.

Adjectif de relation, 1-2, p. 22.

Adjectif d'inhérence, 1-2, p. 22.

Agrégation, 3, p. 163.

Aide-serveur, 3, p. 172.

Allégement, 4, p. 225.

Allusion prestigieuse, 3, p. 155.

Alpinisme, 3, p. 189.

Ambiguiité linguistique, 1-2, p. 11.

Analogies (systèmes d'), 1-2, p. 7.

Analyse contrastive, 1-2, p. 51 .

Analyse frontologique, 4, p. 246.

Analyse morphologique, 1-2, p. 110.

Analyse syntactique, 1-2, p. 64.

Analyse syntagmatique, 1-2, p. 63.

Analyse terminologique, 1-2, p. 61 .

Anglicismes au Québec, 3, p. 180

Anglicismes de fréquence, 1-2, p. 21.

Anglicismes en gestion, 4, p. 248.

Anomalies asystématiques dans les langues, $1-2$, p. 7 .

Appareils électroménagers, 4, p. 234.

Approche analytique de l'exploitation terminologique, $1-2$, p. 101

Approche globale de l'exploitation terminologique, $1-2$, p. 100.

Archaismes (traduction des), 1-2, p. 29.

Argument de tri, 1-2, p. 130.

Arrérages, 3, p. 171.

Arsenal typographique des terminaux, 1-2, p. 126.

Article (rôle dans l'identification du nombre), 1-2, p. 10.

Article et possessif, 1-2, p. 11.

Articles, 4, p. 232.

Articles d'achat sélectif, 4, p. 235.

Articles d'achat spontané, 4, p. 235.

Articles de fantaisie, 4, p. 235.

Articles de nouveauté, 4, p. 234

Articles d'usage / courants, 4, p. 235

Assurance sur la vie, 3 , p. 171

Auprès des clients, 3 , p. 172.

Avantages de l'ordinateur pour l'automatisation des fichiers, 1-2, p. 106 .

Baccalauréat, 3, p. 165 .

Baccalauréat ès arts, 3, p. 169.

Bananier, 1-2, p. 11.

Band microphone, 4, p. 222.

Banque de mots et traducteur individuel, 1-2, p. 114.

Banque de terminologie (U. de M.), 1-2, p. 5-6.

Banque de terminologie de Montréal, originalité, $1-2$, p. 96

Banque d'information, 1-2, p. 95.

Banques de mots, 1-2, p. 73.
Batterie d'accumulateurs, 1-2, p. 77.

Bavard (clichés de comparaison), 3, p. 154.

Bibliographies, 3, p. 187

Bichonnage, 4, p. 228

Bidirectionnel, 4, p. 223.

Bien, 4, p. 231.

Biens auxiliaires, 4, p. 232.

Biens corporels, 4, p. 231.

Biens de consommation, 4, p. 232.

Biens de production, 4, p. 232

Biens d'équipement, 4, p. 233.

Biens d'investissement, 4, p. 233.

Biens directs, 4, p. 232.

Biens durables, 4, p. 233

Biens économiques, 4, p. 232.

Biens fongibles, 4, p. 233.

Biens immatériels, 4, p. 232.

Biens indirects, 4 , p. 232.

Biens matériels, 4, p. 232 .

Biens meubles, 4, p. 231.

Biens mixtes, 4, p. 232.

Biens non durables, 4, p. 233.

Biens non essentiels, 4, p. 235 .

Biens non fongibles, 4, p. 233.

Biens périssables, 4 , p. 233.

Biens semi-consomptibles, 4 , p. 233.

Biens semi-durables, 4, p. 233.

Biens-capitaux, 4, p. 232.

Biologie, 3, p. 189.

Bleuissement, 4, p. 225.

Bois (technique du), 3, p. 190.

Bouchage des trous, 4, p. 226.

Bouée-laboratoire, 4, p. 247.

Bouillie neigeuse / de neige, 4, p. 246.

Boursouflure, 4, p. 226

Cadre, 1-2, p. 9 .

Cafetier, 3 , p. 173

Calques, 4 , p. 258

Canadianismes, $1-2$, p. 13

Centre de documentation, 1-2, p. 96

Cession, 3, p. 171.

Cessionnaire, 3 , p. 171.

Chanci(s), 4, p. 226 .

Changeant (clichés de comparaison), 3, p. 154.

Chantier de débord, 3, p. 174.

Chantier de marchandises, 3, p. 174.

Chapeau mexicain, 4 , p. 211 .

Chasse-neige élevé, 4, p. 245.

Chemisier, 1-2, p. 11 .

Chevier, 1-2, p. 12 .

Chimie, 3, p. 190

CHU, 3, p. 162.

Cinéma, 3 , p. 189

Cliché, 3, p. 155 .

Clichés de comparaison, 3, p. 154.

Clients d'une banque de terminologie, 1-2, p. 98-99.

Cloque, 4, p. 226.

Code de la néologie, 4 , p. 258.

Combustion massique, $1-2$, p. 62 .

Combustion nucléaire, 1-2, p. 62 . 
Compréhension du fond, $1-2$, p. 61 .

Comptabilité de gestion, 4, p. 248 .

Conception de la traduction, 1-2, p. 57

Concurrence en traduction, 1-2, p. 72.

Confection automatique d'un dictionnaire, 1-2,

p. 130.

"Congé sans solde ", 1-2, p. 21.

Consolidation, 3 , p. 171 .

Consomptible, 4, p. 233.

Constantes et variantes, $1-2$, p. 12

Consultation automatique directe, 1-2, p. 109.

Contexte et polysémie, 1-2, p. 11 .

Contextes et définitions en terminologie, 1-2, p. 99.

Contraintes de l'informatique, 1-2, p. 108.

Contrepoint, 3, p. 156.

Contribution, 3, p. 160

Contrôle, $1-2$, p. 79 :

Contrôle de l'information terminologique, 1-2, p. 115.

Coordination des recherches terminologiques, $1-2$, p. 74.

Corrections de l'information en mémoire, 1-2, p. 108.

Coup de foudre, 4, p. 235.

Coupe-circuit, 1-2, p. 77.

Courageux (clichés de comparaison), 3, p. 154.

Coutellerie, 1-2, p. 22

CPEM, 3, p. 162 .

Créancier-garagiste, 3 , p. 171

Critique comparée multilatérale, 1-2, p. 53.

Cuisinier du matin, 3, p. 172

Culture et langue, $1-2$, p. 89

Cuvette, 4, p. 227.

Cycles d'études universitaires, 3, p. 167.

Danger des traductions terminologiques, 1-2, p. 78.

(a Dans le champ ", 3, p. 172.

Débarrasseur, 3, p. 172.

Déclaration des entrées dans une banque de mots, 1-2, p. 127.

Découpage linguistique de la réalité, 1-2, p. 84.

Décrassage, 4, p. 228.

is Décrément logarithmique moyen de l'énergie $», 1-2$, p. 62 .

Dédoublement, 4, p. 227.

Déficiences des dictionnaires bilingues, 1-2, p. 22.

Déficiences des langues naturelles, 1-2, p. 11 .

Définitions (importance en terminologie), 1-2, p. 77.

Délais en traduction, 1-2, p. 65 .

Denrées, 4, p. 231.

Dépoussiérage; 4, p. 228

Désertion temporaire, 3 , p. 156.

Dextre (à), 4, p. 227.

Dictionnaires classiques et dictionnaires automatiques, 1-2, p. 129.

Dictionnaires techniques, 4 , p. 211.

DICAUTOM, 1-2, p. 105.

Directif, 4 , p. 223.

Discrimination des dictionnaires techniques, 4. p. 213 .

Disjoncteur, 1-2, p. 77

Disparités orthographiques, 1-2, p. 128.

Divergence (mathématiques), 1-2, p. 62

Doctorat, 3, p. 163, 167.

Doctorat français, 3, p. 163.
Domaines d'application de l'information terminologique, $1-2$, p. 126.

Doublage, 4, p. 229.

Doubles emplois en recherche terminologique, $1-2$, p. 97.

Drague suceuse, 4, p. 211.

Droit (grades), 3, p. 162.

Droits de tirage spéciaux, 3, p. 156

Dues, 3, p. 163.

Duel, 3, p. 164

Eau (terminologie de 1'), 3, p. 192.

Ecaille en tuiles, 4, p. 227.

Echanges de l'information terminologique, 1-2, p. 112.

Echanges entre usagers au moyen d'une banque de terminologie, 1-2, p. 97.

«e Ecole des gradués », 3, p. 167

Ecole de traduction (orientation), 1-2, p. 71

Economie, 3, p. 189.

Ecran cathodique et banque de mots, 1-2, p. 127.

Edition et banques de mots, 1-2, p. 129.

Effet de pistonnage, 4, p. 246

Effets, 4, p. 231

Effort dûu au vent, 4, p. 247.

Electronique, 3, p. 190.

Electrotechnique, 3, p. 190.

Embu, 4, p. 227.

Emprunt, 3, p. $157 ; 4$, p. 248, 258

Engramme, 1-2, p. 61 .

Enseignement de la traduction, 1-2, p. 65.

Ensemble, 1-2, p. 9.

Entreprise, 1-2, p. 9.

Equivalence, 3, p. 153.

Espace, 1-2, p. 9

Etat fondamental, 1-2, p. 62.

Etat normal, 1-2, p. 62 .

Etoffes, 4, p. 234

Evaluation des traductions, 1-2, p. 93.

Eventue1, 1-2, p. 21

Evolution passée, 4, p. 246.

Explicitations et implicitations, 1-2, p. 11

Explicitations et implicitations (dosage), 1-2, p. 12.

Exploitation conversationnelle d'une banque de terminologie, 1-2, p. 98.

Exploitation d'une banque de mots, 1-2, p. 110.

Facteur de désavantage, 1-2, p. 62.

Facteur de flux neutronique, 1-2, p. 62.

Faisceau de formation, 3, p. 174

Faisceau de réception, 3, p. 174.

Familier, 1-2, p. 21.

Fautes de frappe, 1-2, p. 128

Faux amis, 1-2, p. 9, 21.

Femme de chambre, 3, p. 173

Fiche terminologique, 1-2, p. 126.

Fiche terminologique (éléments), 1-2, p. 101.

Fichier (constitution d'un), 1-2, p. 66.

Fier (clichés de comparaison), 3, p. 154.

Fongible, 4, p. 233.

Formation des terminologues, 1-2, p. 73.

Formation du traducteur, 1-2, p. 66 .

Formation universitaire du traducteur, 1-2, p. 57.

Formations nuageuses, 4 , p. 245.

Forme, élément significatif, 1-2, p. 28.

Fort (clichés de comparaison), 3, p. 154

Français, langue de travail, 1-2, p. 5 .

France (grades universitaires en), 3, p. 162. 
Friteur, 3, p. 173.

Friturier, 3 , p. 173

Garçon de bar, 3, p. 174.

Garçon de lingerie, 3, p. 173.

Garcle-manger, 3, p. 173

Générique et spécifique, 1-2, p. 22.

Générique et spécifique (importance terminologique), 1-2, p. 81 .

Gens, 1-2, p. 8

Géomorphologie, 3, p. 190 .

Gestion, 4, p. 247.

Glaceuse de cols, 3, p. 173.

Glacier, 1-2, p. 11.

Gouvernante, 3, p. 173.

Gouverneur, 1-2, p. 21.

Gracles universitaires, 3 , p. 162

Granmaire comparative, 1-2, p. 17

Granmaire transformationnelle et grammaire traditionnelle, 1-2, p. 60.

Grand (clichés de comparaison), 3, p. 154.

Grec (ressources terminologiques du), 1-2, p. 77.

Gueule de bois, 3, p. 156 .

Hardware, 1-2, p. 70

Harmonisation des notions en terminologie, $1-2$, p. 78

Hiérarchie hôtelière, 3, p. 172

Hippie (phénomène et répercussions linguistiques), $1-2$, p. 15 .

Implicitations et explicitations, 1-2, p. 11

Importance de la traduction, 1-2, p. 58 .

Impôt, 3, p. 160.

Impôt direct, 3, p. 161

Impôt indirect, 3 , p. 161.

Incolérences orthographiques, 1-2, p. 128

Infinitif comme jussif, 1-2, p. 19

Informations terminologiques du domaine juridique, 1-2, p. 108-109.

Informatiaue, 3, p. 181 .

Informatique et formation du traducteur, 1-2, p. 67.

Innocent. 3, p. 154

Insécurité linguistique des locuteurs bilingues, $1-2$, p. 24

Interférences, $1-2$, p. 50

Interprétation, 3, p. 185, 192.

Interrogation de l'ordinateur, $1-2, \mathrm{p}, 128$

Intraduisibilité, 1-2, p. 25.

IUT, 3, p. 164 .

Jaloux (clichés de comparaison), 3, p. 154

is Jongler », 1-2, p. 13.

Justification d'âge, 3 , p. 175 .

Langue administrative, $1-2$, p. 19.

Langue des avis. $1-2$, p. 19

Langue et univers mental, $1-2$, p. 14

Langue, instrument de communication, 1-2, p. 84 .

Langue maternelle et traduction, 1-2, p. 72

Langue, vision du monde, 1-2, p. 26.

Langues naturelles, langues artificielles, 1-2, p. 7 .

Latin (ressources terminologiques du), 1-2, p. 77 .

Laveur de marmites, 3, p. 173.

Lexicographie, 1-2, p. 97.

Lexicologie différentielle, rôle, 1-2, p. 20.

Licence, 3 , p. $163,169$.

Linguistique anglaise, 3 , p. $192 ; 4$, p. 240 .
Linguistique appliquée, 1-2, p. 45.

Linguistique comparative, $1-2$, p. 45.

Linguistique comparative analytique ou synchronique, $1-2$, p. 48.

Linguistique différentielle, 1-2, p. 45.

Linguistique différentielle et traduction, 1-2, p. 17.

Linguistique et traduction, 1-2, p. 7.

Linguistique française, 3, p. 193.

Linguistique mathématique, $1-2$, p. 60 .

Locutions en vedette, 1-2, p. 127.

Long (clichés de comparaison), 3, p. 154.

Longueur des traductions, $1-2$, p. 94.

Luxe, 4, p. 235.

Macrojérôme, 1-2, p. 90

Maîtrise, 3, p. 162.

Marchandise, 4, p. 231

Marchandises sèches », 4, p. 234

Marchandises-capital, 4, p. 232

Masticage, 4, p. 226.

Mat, 4, p. 227.

Matériel d'immobilisation, 4, p. 232

Matière, 4, p. 231.

Matière de remplissage, 4 , p. 226.

Matières intermédiaires, 4 , p. 233

Matières liquides, 4, p. 234.

Matières premières irremplaçables, 4, p. 232

Matité, 4, p. 227

Médecine, 3, p. 189, 190.

Médecine (grades), 3, p. 162.

Mémoire de terminológie, $1-2$, p. 67.

Menus articles, 4 , p. 235.

Mercerie, 4, p. 234.

Métatraduction, 1-2, p. 28.

Météoro'ogie, 3, p. 190

Méthode terminologique, $1-2, \mathrm{p} .111$

Microjérôme, 1-2, p. 90.

Microphone, 4, p. 222.

Microphone à ruban, 4 , p. 222

Microphone à bobine mobile, 4, p. 222

Microphone à capacité, 4, p. 223 .

Microphone à condensateur, 4, p. 223.

Microphone à conducteur mobile, 4, p. 223

"Microphone dynamique ", 4, p. 222

Microphone électrodynamique, $4, \mathrm{~b} .223$.

Microphone électrostatique. 4, p. 223

Modèle d'analyse en traduction, 1-2, p. 83.

Modulation, 3, p. 153

Module lunaire, 3, p. 156

Monovalence du traducteur, 1-2, p. 71 .

Motivation lexicale, $1-2$, p. 22.

Motivation linguistique, $1-2$, p. 26, 77 .

Neige vierge, 4 , p. 246

Néologie, 4, p. 258.

Néologismes, $1-2$, p. 70

Nettoyage, 4, p. 228.

Niveaux de langue, 1-2, p. 29.

Nobélium, 1-2, p. 62 .

Non directionnel, 4, p. 223

Normalisation, 1-2, p. 97.

Notion opérationnelle de traduction, 1-2, p. 89 .

Nouveautés, 4, p. 235.

Objet, 4, p. 232.

Observatoire du français contemporain, 3, p. 194.

Obstacles terminologiques en traduction, 4 , p. 211.

Office, 3, p. 174 
Officière, 3, p. 174.

Omnidirectionnel, 4, p. 223.

On (emploi affectif), 1-2, p. 31 .

Onde acoustique, 4, p. 223 .

Onde sonore, 4, p. 223 .

Options stylistiques, 1-2, p. 19.

Organisation commerciale, 4, p. 248

Organisation professionnelle (rôle et orientation), $1-2$, p. 72 .

Orthographe américaine et orthographe anglaise, 1-2, p. 128.

"Padding ", 4, p. 212

Paramètre de ralentissement, 1-2, p. 62.

Paresseux (clichés de comparaison), 3, p. 154.

Particules verbales anglaises, 1-2, p. 16.

Passif (anglais et français), 1-2, p. 15, 19, 30.

Patenaude, Paul, 4, p. 260.

Peau de crapaud, 4 , p. 228.

Pétrolier, 1-2, p. 11.

Pharmacie (grades), 3, p. 162

Phonétique anglaise, 1-2, p. 18

Photographie d'amateur, 3, p. 189.

Phraséologie terminologique, 4, p. 213.

Pilori (au), 3, p. 175.

Plage, 4, p. 228.

Plasma, 1-2, p. 62.

Pluie verglaçante, 4, p. 246

Pluriels irréguliers, $1-2$, p. 10.

Pointillage, 4, p. 229.

Polymorphies dans les langues naturelles, 1-2, p. 8.

Polysémies dans les langues naturelles, 1-2, p. 8-9.

Pompage, 4, p. 246.

Pont roulant, 1-2, p. 107.

Pont thermique, 1-2, p. 107

Porte à porte, 3, p. 171.

Portefeuille d'agent, 3, p. 172.

Portefeuille d'assuré, 3 , p. 172 .

Portefeuille de contrats, 3, p. 172.

Porteur, 3, p. 172.

Possibilité de la communication, 1-2, p. 85.

Possibilité de la traduction, 1-2, p. 85.

Poudrerie, 4, p. 245.

Préposé, 3, p. 173.

Prestation d'appoint, 3, p. 172.

Préventeur, 3, p. 175.

Prévention des incendies, 3, p. 188.

Préventionniste, 3 , p. 175 .

Prime de risque, 3 , p. 172.

Prime d'épargne, 3, p. 172.

Processus de traduction, 1-2, p. 60.

Prochainement, 1-2, p. 22.

Procureur général, 3, p. 156.

Production, 4, p. 232.

Produit, 4, p. 231.

Produits industriels, 4, p. 233.

Produits intermédiaires, 4, p. 233.

Projection conique équivalente de Lambert, 4, p. 247.

Pronom possessif anglais, 1-2, p. 11.

Propre (clichés de comparaison), 3, p. 154.

Propriétés spécifiques des langues naturelles, $1-2$, p. 7.

Pseudo-scientifisme de la langue courante, 1-2, p. 69.

Puissance sur l'arbre, 1-2, p. 78.

Québec (grades universitaires aul), 3, p. 165.
Rapide (clichés de comparaison), 3, p. 154.

Radio (préfixe), 1-2, p. 64.

Rapports internes du texte traduit, 1-2, p. 61.

Recherche et traduction, 1-2, p. 67.

Recherches en linguistique contrastive, 1-2, p. 46.

Recherches terminologiques et traduction, 1-2,

p. 106.
Redondances dans les langues naturelles, 1-2, p. 10.

Réduction à une forme normale des entrées, $1-2$, p. 128.

Régénération, 4, p. 229.

Régionalismes en terminologie, 4, p. 214.

Relais de mesure, 1-2, p. 78.

* Relais extranomes", 1-2, p. 78.

"Relais intranomes", 1-2, p. 78.

Relais tout ou rien, $1-2$, p. 78

Relation auteur-traducteur-utilisateur, 1-2, p. 86.

Rentoilage, 4, p. 229.

Repeint, 4, p. 229.

Repentir, 4, p. 229

Répertoires, 3, p. 191.

Repiquage, 4, p. 229.

Ressources virtuelles des langues à l'égard de

la communication, 1-2, p. 83.

Restauration des peintures, 4, p. 224.

Résulter, 1-2, p. 21.

Retraite (régime de), 3, p. 174.

Retraite à l'âge normal, 3, p. 175.

Retraite anticipée, 3, p. 174 .

"Retraite normale ", 3, p. 175.

Rigatini, 4, p. 230.

Route, 1-2, p. 21.

Routier, 1-2, p. 11.

Rusé (clichés de comparaison), 3, p. 154

Salaire cotisable, 3, p. 174.

Satellite, 1-2, p. 9 .

Science de la traduction, $1-2$, p. 58,66 .

Sélection des dictionnaires techniques, 4 p. 212.

Senestre (à), 4, p. 227

Sens interdit, 1-2, p. $19 ; 3$, p. 156

Services terminologiques aux techniciens et non-techniciens, 1-2, p. 98-99.

Servitudes grammaticales, 1-2, p. 19.

"Set de chambre », 1-2, p. 23.

(ce Simultiste », 3, p. 185 .

Situation extralinguistique, 1-2, p. 11.

* Slush », 4, p. 246.

Société des traducteurs du Québec, 3, p. 195.

Sociologie, 3, p. 189.

Software, $1-2$, p. 70.

Solde (f.), 1-2, p. 21.

Solidarité professionnelle, 4, p. 259.

«Sollicitation à froid », 3, p. 171.

Solution des problèmes non numériques par l'informatique, 1-2, p. 105.

Sondage, 4, p. 230

Soucoupe volante, 3, p. 156.

Source de l'information terminologique et critère de validité, $1-2$, p. 80

Spécialisation des terminologues par langue, $1-2, p .107$

Spécialisation du traducteur, 1-2, p. 68 .

Spécialités (langue des), 1-2, p. 70.

Stages, 1-2, p. 67. 
Structuralisme et traduction, 1-2, p. 59 . Structure associative d'une banque de terminologie, $1-2$, p. 100

Structure grammaticale, 1-2, p. 89

St.ucture mentale, $1-2$, p. 14

Structures élaborees, $1-2$, p. 64

Structures et sens des énoncés, $1-2$, p. 18

Structures profondes, $1-2$, p. $64,89$.

Substance (catégorie) 1-2, p. 27.

Sur le terrain, 3 , p. 172.

Synonymes (traitement des), 1-2, p. 127.

Synonymie technique (exemple de), 1-2, p. 98.

Syntagmes (types de), 1-2, p. 63.

Table chauffante, 4 , p. 230

Table de mortalité, 3, p. 172 .

Tarifs de rémunération, $1-2$, p. 72

Taux d'épuisement, 1-2, p. 62

Taxe, 3, p. 160 .

Team, 1-2, p. 125 .

Téléphoniste du service à l'étalage, 3, p. 174.

Terminologie, 1-2, p. 125

Terminologie (définition), 1-2, p. 75 .

Terminologie des grades universitaires, 3 , p. 162.

Terminologie et lexicographie, 4 , p. 215.

Termino'ogie et traduction, 1-2, p. 23

Terminologie juridique, 1-2, p. 109.

Terminologues (formation des), 1-2, p. 73 .

Textes originaux en recherche terminologique, $1-2$, p. 107

Théologie, 3, p. 189 .

Tissus, 4, p. 234.

Traducteur et banque de mots, 1-2, p. 105

Traduction (définition de la), 1-2, p. 14

Traduction (importance de la), 1-2, p. 58

Traduction (nature), $1-2$, p. 25

Traduction automatique, 3, p. 192.
Traduction des grades universitaires, 3, p. 170-171.

Traduction électronique, 1-2, p. 96.

Traduction et lexicographie, 4, p. 215.

Traduction et linguistique, $1-2$, p. 7 .

Traduction et linguistique moderne, 1-2, p. 52.

Traduction et terminologie, 1-2, p. 76 .

Traduction humaine, 3, p. 192.

Traduction littéraire, 1-2, p. 29.

Traduisibilité et intraduisibilité, 1-2, p. 25.

Transcodage et traduction, $1-2, \mathrm{p} .85$.

Transfert, 4 , p. 230

Transferts négatifs, $1-2$, p. 49.

Translittération, $1-2$, p. 126 .

Transport supersonique, 3 , p. 156.

Triage, 3, p. 174

"Trowal $», 4$, p. 246

Unidirectionnalité de la traduction, 1-2, p. 54.

Unidirectionnel, 4, p. 223.

Unité de traduction, 1-2, p. 89

Univers mental, 1-2, p. 14 .

Universalité de la traduction, 1-2, p. 86

Université de Montréal, rôle en traduction, $1-2$, p. 5 .

Universités anglophones du Québec (grades), 3 , p. 166

Universités francophones du Québec (grades) 3, p. 167

Utilisation électronique de la Banque de mots, $1-2$, p. 95.

Variantes (connotation sociologique des), 1-2, p. 14.

Variantes (divers types), 1-2, p. 12

Variantes canadiennes-françaises, 1-2, p. 13.

Variante/constante (rapport), 1-2, p. 16.

Verbe de mouvement à distinguer du verbe de destination, $1-2$, p. 20

Verbe « vectoriel ", $1-2$, p. 20 